\title{
Access Point Selection Strategy in IEEE802.11e WLAN Networks
}

\author{
Shojiro Takeuchi*, Kaoru Sezaki ${ }^{\dagger}$ and Yasuhiko Yasuda* \\ ${ }^{*}$ Grad. School of Science and Engineering, Waseda University \\ 3-4-1 Okubo, Shinjyuku-ku, Tokyo, 169-8555 Japan \\ Email: shojiro,yasuda@yasuda.comm.waseda.ac.jp Telephone: +81 (3) 5286-3388 \\ $\ddagger$ Center for Spatial Information Science at University of Tokyo \\ Komaba 4-6-1, Meguro-ku, Tokyo, 153-8505 Japan \\ Email: sezaki@iis.u-tokyo.ac.jp Telephone: +81 (3) 5452-6268
}

\begin{abstract}
IEEE802.11 WLAN (Wireless LAN) has been widely used in enterprise and public space such as air port. In these large WLAN networks, RRM (Radio Resource Management) is necessary for the efficient use of radio resource and also for load balancing among APs (Access Point) Since, in IEEE802.11, STA (Station) has the right to select an AP with which it will associate, AP selection mechanism implemented in STA is important for RRM. This paper proposes an AP selection mechanism, called HRFA (High-Rate First Association) in order to achieve load balancing and the efficient use of radio resource. Furthermore, IEEE802.11e is currently standardizing a MAC protocol to provide QoS (Quality of Service) in MAC layer and support real-time traffic over WLAN. In order for WLAN to be more widely used and to provide QoS in WLAN networks, the functionalities of IEEE802.11e have to be provided in WLAN devices. Our proposed HRFA can be applied to IEEE802.11e WLAN and can be implemented without any modifications in IEEE802.11 and 802.11e standard. Therefore it is useful from implementation-cost and compatibility point of views. Simulation results show that HRFA can efficiently ultilize radio resource and also achieve load balancing in IEEE802.11e WLAN networks.
\end{abstract}

\section{INTRODUCTION}

Nowadays, IEEE802.11 [1] WLAN is mainly used for Internet access, but real-time application like VoIP (Voice over IP) and video conference are identified as next killer applications for WLAN. Since these applications require distinct specific features, such as delay sensitivity or bandwidth requirement, it is desired to support differentiation services in IEEE802.11. Therefore, IEEE 802.11e working group is now discussing new 802.11 MAC protocol, which provides QoS [2]. The 802.11e HCF (hybrid coordination function) provides both a contention-based channel access, called EDCA (enhanced distributed channel access), and a controlled channel access, referred to as HCCA (HCF controlled channel access). In this paper we focus on the EDCA. In order for WLAN to be more widely used and to support QoS in WLAN networks, the functionalities of $802.11 \mathrm{e}$ have to be provided in WLAN devices.

WLAN is currently used in office and public area such as hotel and airport. In these large WLAN networks, RRM is important for the efficient use of wireless bandwidth. For example, since three channels (channel number 1, 6, 11) can be used simultaneously in IEEE802.11b [3], APs have to be

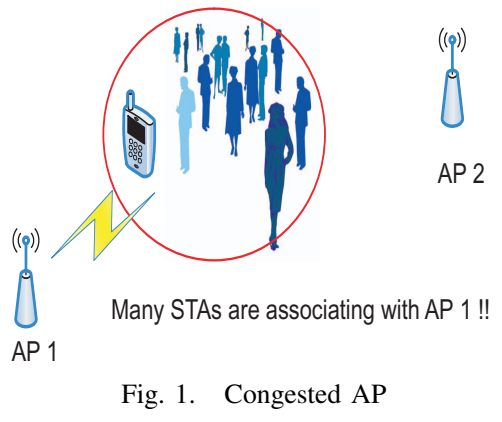

deployed to minimize overlaps between the same channels and to reduce interferences between STAs using the same channel[4]. Besides AP deployment a stratery, strategy for STAs to select an AP with which they will associate is also much important in large WLAN networks. Suppose that there are two APs as shown in Fig.1. If STAs have no strategy, they may associate with one of the APs. As a result, throughput in an AP will decline due to congestion even though radio resource in another AP is available. To overcome such problem presented in Fig.1, there are two approaches. One is centralized way proposed in [5]. In [5], a server is used to control STAs' association and works as admission controller. In fact, admission control is supported in IEEE802.11e, but it's difficult for admission controller provided in IEEE802.11e to be used for non real-time traffic because such kind of traffic has no periodicity. Moreover, IEEE802.11 does not standardize any specific servers to control STAs' association and thus if those servers are required, it is difficult to maintain compatibility among WLAN devices provided from different vendors. In fact, IEEE802.11 specifies only procedures required before a STA connects to an AP [1]. For example, when Passive Scan takes place, a STA receives beacon frames transmitted from APs and decides an AP with which it will associate. That is, STAs have the right to select an AP in IEEE802.11 standard [1]. In addition to limitations in the current standard, if specific servers are needed to control STAs' association, latency taken during the $\mathrm{HO}$ (Hand-Over) procedure will increase. To reduce latency in $\mathrm{HO}$, it is better for STA to select an AP. From these reasons, decentralized approaches were proposed in [8] and [9] and they focused on AP selection mechanisms implemented 
in STAs. This paper discusses and proposes an AP selection mechanism for load balancing and the efficient use of radio resource.

In [8] and [9], AP selection mechanisms were proposed for load balancing among APs. Their proposed algorithms considered RSSI (Received Signal Strength Indicator) and the number of STAs connecting to an AP, and also took into account only BE (Best Effor) traffic. However, in 802.11e WLAN networks, traffic types and their traffic loads should be considered rather than the number of STAs. Therefore, it is difficult to apply their algorithms to IEEE802.11e networks. Moreover, they assumed that STAs could use the same transmission rate even when they connected to APs that were very far from it. However, in general, if a STA communicates with an AP that is far from it, it needs to use low transmission rate. Therefore, taking into account only the number of STAs for load balancing results in increasing the number of STAs that use low transmission rate. Consequently radio resource is inefficiently utilized and throughput of WLAN networks declines. To overcome problems presented above, this paper proposes an AP selection mechanism, called HRFA. It considers channel load in an AP for load balancing and also transmission rate that a STA uses to communicate with an AP for the efficient use of radio resource. Furthermore HRFA considers types of traffic the STA has so that it can be applied to IEEE802.11e networks. In fact, HRFA can be implemented without any modifications in IEEE802.11 and $802.11 \mathrm{e}$ standard, and so it is fully compatible with IEEE802.11 standard. The remainder of this paper is organized as follows. The next section introduces IEEE 802.11e EDCA and admission control. In Section 3, realated researches and their problems are presented. Section 4 shows our proposed algorithm, HRFA. Performance evaluations of our proposal are carried in the section 5, comparing with other schemes. Finally in section 6 , we present the conclusion.

\section{A. $E D C A$}

II. IEEE802.11E

EDCA provides differentiated and distributed channel access to the WM (Wireless Medium) based on 8 different UPs (user priorities). The EDCA mechanism defines four ACs (access categories) to support differentiated channel access. The mapping from UPs to ACs is defined in [2]. Differentiated channel access is achieved through varying the amount of time a station would sense the channel to be idle and the length of CW (Contention Window) for a backoff. Four ACs use different values of AIFS (arbitration inter frame space), the minimum $\mathrm{CW}$ size and the maximum $\mathrm{CW}$ size. In this paper, for $\mathrm{AC} k(0 \leq k \leq 3)$, the minimum $\mathrm{CW}$ size is $C W_{\min }[k]$, the maximum CW size is $C W_{\max }[k]$, and the arbitration inter frame space is $A I F S[k]$. Further the arbitration inter frame space number is $A I F S N[k]$. The relation between $A I F S[k]$ and $A I F S N[k]$ is as follows.

$$
\text { AIFS }[k]=\text { AIFSN }[k] \times \text { slotTime }+ \text { SIFSTime }
$$

In EDCA, real-time traffic in one $\mathrm{AC}$ has a smaller, $A I F S N[k], C W_{\min }[k]$, and $C W_{\max }[k]$ than non real-time trafifc in another AC, it can have a better chance to access the WM earlier that non real-time traffic.

\section{B. Admission Control under EDCA}

This subsection shows admission control method under EDCA presented in [2].

If admission control is needed for an AC, a STA has to send an ADDTS (add traffic stream) request frame to the AP. The ADDTS request contains TSPEC (traffic specification), such as mean data rate, nominal MSUD size, delay bound and etc.. When the AP receives an ADDTS request, it makes a determination as whether to accept or deny the request. If it accepts the request, it calculates from information conveyed in the request the amount of time for requested traffic to access the WM per one second, which is called medium_time. Even though any algorithms can be used for deriving medium_time, a recommended procedure is presented in [2]. After calculating it, the AP sends to the STA an ADDTS response frame, which contains derived medium_time. On receipt of the response from the AP, the STA adds medium_time to a local variable, admitted_time, if the request is admitted. It also has another local variable, used_time. The used_time presents how long the STA has accessed the WM. Using the admitted_time and the used_time, the STA controls the channel access to the WM. The used_time are updated after each successful or unsuccessful MPDU (re) transmission attempt as follows,

$$
\text { used_time }=\text { used_time }+ \text { MPDUExchangeTime }
$$

Further, at seconds interval,

$$
\text { used_time }=\max ((\text { used_time }- \text { admitted_time }), 0)
$$

MPDUExchangeTime is the duration needed to transmit one data frame, considering the duration of SIFS or ACK transmission [2]. If used_time reaches or exceeds the admitted_time, the corresponding AC cannot transmit any frames using its EDCA parameter-set until the used_time is reset. Admission control mechanism is supposed to be applied to real-time traffic, because non real-time traffic does not have in general periodicity and it is difficult to use admission control mechanism.

\section{RELATED WORKS}

RSSI is a metric widely used to decide an AP with which a STA will associate. In this mechanism, the STA receives beacon frames from APs through scanning procedure and monitors RSSI of received frames. At the end of the scanning procedure, it selects an AP with maximum RSSI. In general, if the STA selects an AP with larger RSSI, it can use higher transmission rate and can efficiently utilize radio resource. Consequently the AP can accomodate more traffic and throughput in the AP becomes high [10]. However, the consideration of only RSSI results in traffic congestion in an AP as shown in Fig.1. In order to achieve load balancing among APs, in [8] and [9], AP selection mechanisms, which considered the number of associating STAs, were proposed. In [9], the following metric was proposed.

$$
\text { Score }_{i}=\frac{1-P_{i}}{N_{i}}
$$


$P_{i}$ denotes PER (Packet Error Rate) and $N_{i}$ is the number of associating STAs with AP $i(0 \leq i \leq L-1)$. In [9], the way to calculate PER was not presented, but since it showed that PER was calculated from RSSI, PER is considered as the same as RSSI. After calculating Eq.(3) for all APs with which a STA can communicate, an AP with maximum value of Score $_{i}$ is selected. In [8] and [9], all STAs were assumed to transmit only BE traffic. However, in fact, channel load in the AP may be low, even if the number of associating STAs is large. Therefore, it is better to consider channel load in an AP. And, in 802.11e networks, types of traffic (e.g. real-time or non real-time) have to be considerd when channel load is taken into account. Since in 802.11e networks real-time traffic have better chance to access the WM than non real-time traffic, it is better for a STA with real-time traffic to connect to an AP whose real-time traffic load is low even if its non real-time traffic load is slightly high. Moreover, algorithms proposed in [8] and [9] select an AP that is far from a STA and the STA has to use low transmission rate even if other APs that are near to the STA can accomodate its traffic. As a result, radio resource is inefficiently utilized and throughput in an AP declines. From these observations, algorithms presented in [8] and [9] possibly degrade the performance of WLAN networks even though they tried to achieve load balancing. In the next section, we present our proposed algorithm, HRFA.

\section{A. Overview of HRFA}

$$
\text { IV. HRFA }
$$

This subsection shows an AP selection strategy in HRFA. We assume that in 802.11e networks admission control is taken for real-time traffic (traffic belonging to AC3 and AC2). The strategy of HRFA is to use as high transmission rate as possible in order to efficiently utilize radio resource, and only after traffic load in an AP is high, it selects an AP whose traffic load is low. Therefore HRFA is focus on transmission rate as one of metrics.

Here, load banancing strategy taken by HRFA is presented. The strategy is classified into two types, one for real-time traffic and another for non real-time traffic. First, we show load balancing strategy for real-time. When a STA having real-time traffic selects an AP, the traffic must be accepted by admission controller implemented in the AP. If it can be accepted, realtime traffic load in the AP has to be considered since realtime traffic can more often access the WM than no real-time traffic. Therefore, in HRFA, a STA with real-time traffic selects an AP whose real-time traffic load is low. However, if there is little difference in real-time traffic load among APs, the STA selects an AP, with which it can communicate using higher transmission rate, in order to efficiently utilize radio resource. Next, load balancing strategy for non real-time traffic is presented. When channel load in an AP is low, a STA having non real-time traffic can have more opportunity to access the WM. Therefore, the STA with non real-time traffic selects an AP whose channel load is low. However, as similar to the strategy for real-time traffic, if there is little difference in channel load among APs, the STA selects an AP with which it can communicate at higher transmission rate.

\section{B. Algorithm of HRFA}

This subsection shows a detailed algorithm of HRFA. HRFA considers the following metrics.

- transmission rate

- channel load in an AP

- real-time traffic load in an AP

Transmission rate used to communicate with an AP is determined by LA (Link Adaptation) algorithm implemented in STAs. For example, in [11], transmission rate is decided based on the value of RSSI. Therefore this paper also assumes that transmission rate is decided based on RSSI. However, in fact, any LA algorithms are applicable to HRFA. Channel load and real-time traffic load in an AP are announced to STAs by QBSS load element in beacon frame [2]. Therefore, if a STA executes a scanning procedure, it can get these information [1], [2].

First, the case where a STA with real-time traffic selects an AP is presented. As mentioned in the previous subsection, real-time traffic load in an AP and transmission rate have to be taken into account for AP selection mechanism of STAs with real-time traffic. In HRFA a STA having real-time traffic calculates Eq.(4) and selects an AP wiht maximum value of Score $R T_{i}$.

$$
\text { Score } R T_{i}=A A C_{i} \times R_{i}
$$

$A A C_{i}$ shows Available Admission Capacity, which is announced by QBSS load element. When the number of realtime traffic accomodated in $\mathrm{AP} i$ is $n_{i}, A A C_{i}$ presents the remaining channel time for real-time traffic per a second as shown in Eq.(5).

$$
A A C_{i}=1-\sum_{j=0}^{n_{i}-1} \text { medium_time } j
$$

As explained in section 2, medium_time ${ }_{j}$ denotes time allocated to flow $j$ by the AP. In Eq.(4), $R_{i}$ is a weight factor determined based on transmission rate. As shown in Eq.(6) and (7), it is expressed by using time, $T_{r_{h}}$, needed to transmit at transmission rate of $r_{h}$ a data frame whose payload size is $S$. When calculating $T_{r_{h}}$, headers both in physical and MAC layer are considered. $T_{\max }$ denotes time needed to transmit a data frame at minimum transmission rate. We assume that in Eq.(7) the number of transmission rates supported in a STA is $H$ and that in Eq.(6) $r_{h}$ is selected for the STA to communicate with AP $i$

$$
\begin{gathered}
R_{i}=\frac{T_{\max }}{T_{r_{h}}} \\
T_{\max }=\max \left\{T_{r_{h}} \mid 0 \leq h \leq H-1\right\}
\end{gathered}
$$

Suppose that we use $802.11 \mathrm{~b}$ and can use three transmission rates, $2.0,5.5$, and $11.0 \mathrm{Mbps}$. If we choose 1024 bytes as the value of $S$, we can calculate $R_{i}$ as about 1.0, 2.6, and 4.6 for 2.0, 5.5, and 11.0Mbps, respectively. When we use $A A C_{i}$ and $R_{i}$ in Eq.(4), we can consider influence of transmission rate on the remaining capacity to accomodate real-time traffic. Moreover, in Eq.(4), the current real-time traffic load is not 
considered but the remaining capacity is taken into account because of the following reason. Suppose that STA $a$ can communicate with AP $l$ and $m$ using transmission rate, 11.0 and 2.0Mbps, respectively, and that the current real-time traffic load in AP $l$ is 4.7 times larger than one in AP $m$. In this case, if we use in Eq.(4) the current real-time traffic load in place of remaining capacity for real-time traffic, STA $a$ selects the AP $m$ and uses low transmission rate even if there is a lot of remaining capacity to accomodate real-time traffic in AP $l$. Thus, radio resource is inefficiently utilized. Therefore, to avoid the inefficient use of radio resource, we consider the remaining capacity for real-time traffic in Eq.(4). Taking into account the remaining real-time traffic capacity and transmission rate the Eq.(4) encourage STAs with realtime traffic to select an AP with which it can communicate at higher transmission rate. Moreover, only after real-time traffic load in an AP is high, Eq.(4) allows the STA to select an $\mathrm{AP}$, with which it communicates at low transmission rate, for the purpose of load balancing. Note that even if the value of $S$ core $R T_{i}$ is the highest, a STA cannot associate with AP $i$ and connects to other APs when admission controller implemented in the AP does not accept its request.

Next, we explain an algorithm for non real-time traffic. A STA having non real-time traffic calculates the Eq.(8) and selects an AP with maximum value of $S$ core $N R T_{i}$.

$$
\text { ScoreNRT } T_{i}=\left(256-C L_{i}\right) \times R_{i}
$$

$C L_{i}$ indicates channel load in AP $i$ given from channel utilization field in QBSS load element [2]. It is measuered at the AP and presented as a value ranging from 0 to 255 . As mentioned in the previous section, non real-time traffic can have a better chance to access the WM when it communicates with an AP whose channel load is low. Therefore, in Eq.(8), we consider channel load as one of metrics. And, as similar to the algorithm presented for real-time traffic, in order to efficiently utilize radio resource, waight factor $R_{i}$ and reaminging channel load are considered in Eq.(8). As a result, Eq.(8) encourages STAs with non real-time traffic to associate an AP with which they can communicate at high transmission rate. If we subtract $C L_{i}$ from 255 in Eq.(8), $S$ core $N R T_{i}$ will become 0 and transmission rate cannot be considered in Eq.(8). Therefore we subtract $C L_{i}$ from 256 in Eq.(8).

Although we separately explain algorithms for real-time and non real-time, if a STA has both real-time and non real-time traffic, it uses the algorithm for real-time traffic. Moreover, since channel load and wireless link condition changes over time, $\mathrm{HO}$ is necessary. However, since HRFA is the algorithm to select an AP, it is applicable to $\mathrm{HO}$ algorithms proposed in [7] or [9].

\section{Performance Evaluation}

We implemented our proposed algorithm in NS-2 [12] and performed simulations. We assume that both AP and STA operate with IEEE802.11b[3], basic rate is $2.0 \mathrm{Mbps}$, and data rate is choosen from $2.0,5,5$, and $11.0 \mathrm{Mbps}$. For simplicity, data rate is determined by distance between the STA and AP.

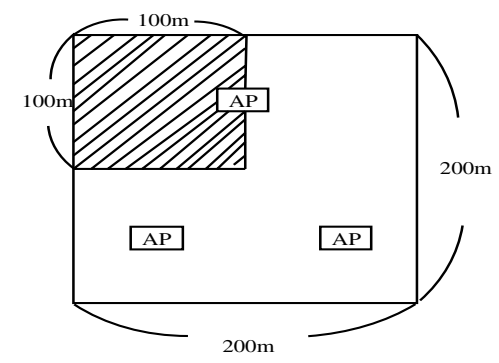

Fig. 2. Simulation Area

STAs within $60 \mathrm{~ms}$ from AP use $11.0 \mathrm{Mbps}$, 5.5Mbps within $120 \mathrm{~ms}$, and $2.0 \mathrm{Mbps}$ within $200 \mathrm{~ms}$, respectively. For each AC, we have the following parameters [2]: $C W_{\max }[0]=1023$, $C W_{\min }[0]=31 ; C W_{\max }[1]=1023, C W_{\min }[1]=31$; $C W_{\max }[2]=31, C W_{\min }[2]=15 ; C W_{\max }[3]=15$, $C W_{\min }[3]=7 ; \operatorname{AIFS}[0]=7, \operatorname{AIFS}[1]=3, \operatorname{AIFS}[2]=2$, $A I F S[3]=2$. Simulation area is $200 \mathrm{~m} \times 200 \mathrm{~m}$. Three APs are placed in $(42,50),(100,150)$, and $(158,50)$, respectively. These APs operate in different channels and so they do not interfere with each other. Two types of deployment of STAs are considered. First STAs are uniformly distributed in the simulation area (Case1). Next they are deployed in a place shaded in Fig.2 (Case2). For both STA deployments, simulations are performed. As simulation traffic, voice, video, and data traffic are used. Each voice flow is $83.2 \mathrm{Kbps}$, which is generated by a constant interval, $20 \mathrm{~ms}$ and has a fixed payload size of 208 bytes. This flow corresponds to G.711coded VoIP [6]. Each video flow is $770 \mathrm{Kbps}$, which is generated by a constant interval, $13.33 \mathrm{~ms}$ and has a fixed payload size of 1280 bytes. As background traffic, UDP traffic is $800 \mathrm{Kbps}$, which is generated by a constant interval, 10 $\mathrm{ms}$ and has a fixed payload size of 1024 bytes. In each simulation run, simulation runs for 200s Simulation traffic is generated in the interval from 0 s to $100 \mathrm{~s}$. We use simulation outputs in the interval from 100s to 200s in each simulation. We evaluate throughput for all voice, video, and data traffic, and also evaluate delay of voice and video traffic. When we generate simulation traffic, first we randomly select a STA and next traffic which the STA transmits is choosen from voice, video, and data traffic. Finally when simulation time is $100 \mathrm{~s}$, there are the same number of voice, video, and data traffic in each simulation. That is, if flow-set is 5 in simulation results in the following subsection, it means there are 5 voice, 5 video, and 5 data flows. In each simulation, HRFA is compared to two AP selection algorithms. One of them is AP selection algorithm taking care only about RSSI (called RSSI). Another is one considering the number of associating STAs, which was proposed in [9] (called NumSTA). In the following simulations, all AP performs admission control and minimum physical rate is set to 5.5Mbps. Therefore, reagardless of AP selection algorithms, a STA having real-time traffic cannot associate with an AP with which it communicate at 2.0Mbps.

\section{A. Simulation Results in Case1}

Fig. 3 and 4 show average throughput of voice and video traffic, and Fig.6 and 7 present average delay of voice and 


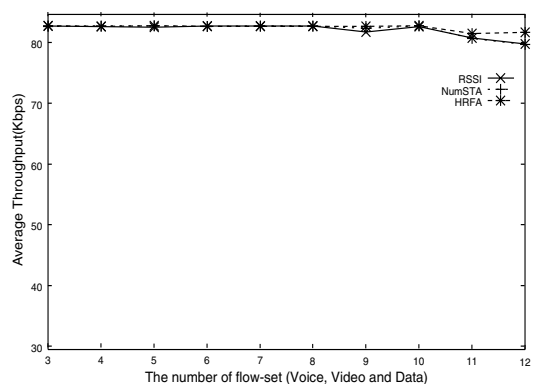

Fig. 3. Average throughput of voice traffic in Case1

video traffic. In case where STAs are uniformly deployed in the simulation area, the number of traffic and traffic types are also in a way distributed in the area. Therefore, with reagard to throughput and delay of voice and video traffic, there is litte difference among RSSI, NumSTA, and HRFA. This is because real-time traffic can have better chance to access the WM under 802.11e EDCA. Thus throughput and delay of realtime traffic are maintained. However, in case of NumSTA, throughput of video traffic declines and also its delay increases when the number of traffic increases. NumSTA distributes the number of associating STAs but does not take into account traffic types and load. And, using NumSTA, the number of data traffic transmitted at low transmission rate increases. Furthermore, even if STAs having real-time traffic can connect to an AP using transmission rate of $11.0 \mathrm{Mbps}$, they may associate with other APs with which they communicate at $5.5 \mathrm{Mbps}$. As a result, radio resource is inefficiently utilized and throughput and delay of video traffic is degraded when there are much traffic in the simulation area.

Fig.5 shows average throughput of data traffic. Especially in NumSTA, throughput of data traffic is low. As mentioned above, NumSTA distributes only the number of associating STAs and so the number of traffic transmitted at low transmission rate is increased. Therefore, the inefficient use of radio resource result in low throughput of data traffic. Since RSSI and HRFA select an AP with which STAs can communicate at high transmission rate, radio resource is efficiently utilized. But in case where only RSSI is considered, traffic congestion happens in a specific AP. On the contrary, STAs with HRFA can associate with APs whose channel load is low when channel load increases in an AP. Therefore, in HRFA, radio resource is efficiently used and load balancing is also achieved. As a result, throughput of data traffic in HRFA is higher than in RSSI.

\section{B. Simulation Results in Case2}

This subsection shows simulation results in case of simulations performed in Case2 scenario. Fig. 8 and 9 present average throughput of voice and video traffic, and Fig.11 and 12 show average delay of voice and video traffic, respectively. When using 802.11e, real-time traffic is given a better chance to access the WM. Therefore in case where traffic load is low, throughput of voice and video traffic is not degraded even in RSSI and NumSTA. However, when traffic load is high, throughput of voice and video in RSSI and NumSTA

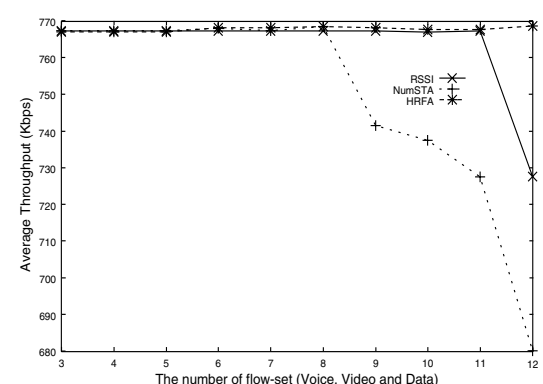

Fig. 4. Average throughput of video traffic in Case1

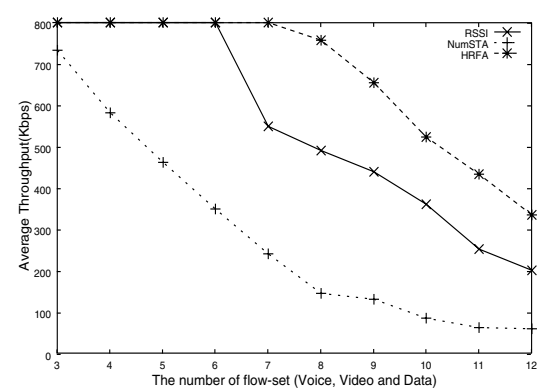

Fig. 5. Average throughput of data traffic in Case 1

declines and accordingly their delay increase. Since RSSI does not consider load balancing, traffic load becomes high in a specific AP and contentions between real-time flows or between real-time and non real-time flows increase. Consequently throughput of voice and video declines even when 802.11e is used. In case of NumSTA, since its load balancing strategy does not care about traffic types, real-time traffic will be possibly congested in a specific AP. Thus, throughput of real-time traffic is degraded. Furthermore, in NumSTA, STAs not only with real-time but also with non real-time traffic may communicate with an AP, which is far from them, at low transmission rate. As a result, radio resource is inefficiently

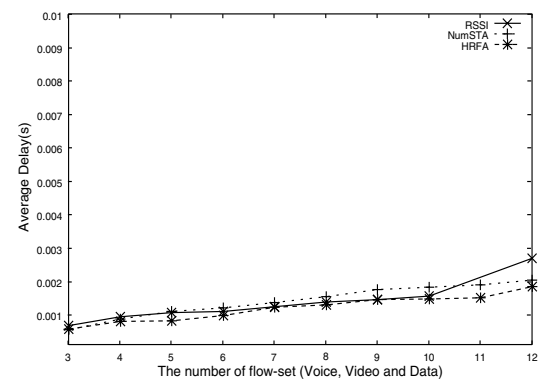

Fig. 6. Average delay of voice traffic in Case1

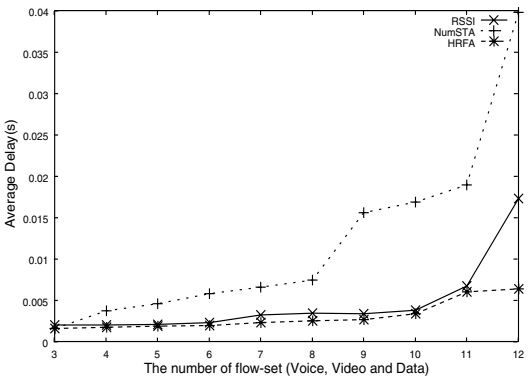

Fig. 7. Average delay of video traffic in Case 1 


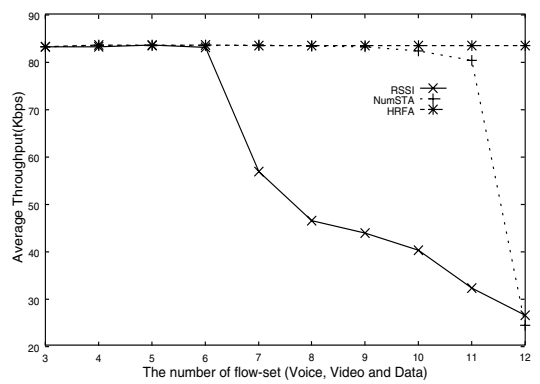

Fig. 8. Average throughput of voice traffic in Case2

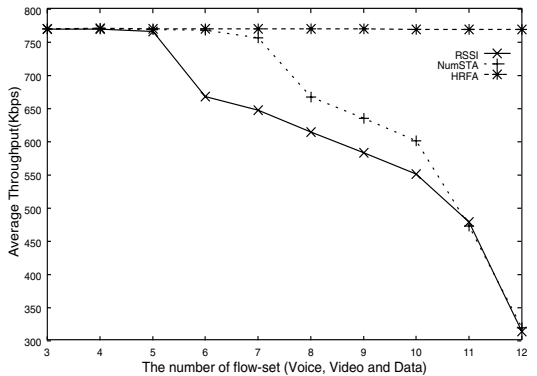

Fig. 9. Average throughput of video traffic in Case2

used and throughput of real-time traffic is degraded when traffic load is high. Accordingly its delay increases. On the contrary, in HRFA, throughput and delay of real-time traffic are maintained even when traffic load is increased. This is due to the fact that HRFA encourages STAs to connect to an AP with which they can communicate at high transmission rate and it takes them to other APs only after traffic load is high in an AP. That is, HRFA achieves the efficient use of radio resource and load balancing. Furthermore, since load balancing strategy in HRFA considers traffic types, real-time traffic is not congested in an specific AP.

Fig.10 presents average throughput of data traffic. Since in HRFA radio resource is effieciently utilized and load balancing is also achieved, throughput of data traffic is higher than other methods. When traffic load is increased, difference between througput in HRFA and RSSI becomes little. This is due to the fact that when traffic load is high, throughput of real-time traffic in RSSI declines and on the other hand one of data traffic is maintained to some extent.

\section{CONCLUSION}

In this paper, we proposed HRFA as an AP selection mechanism implemented in STAs. It considers transmission

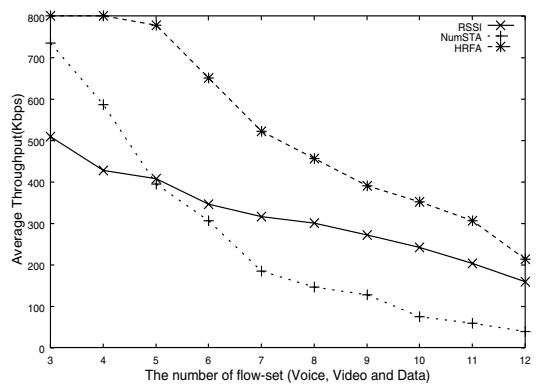

Fig. 10. Average throughput of data traffic in Case2

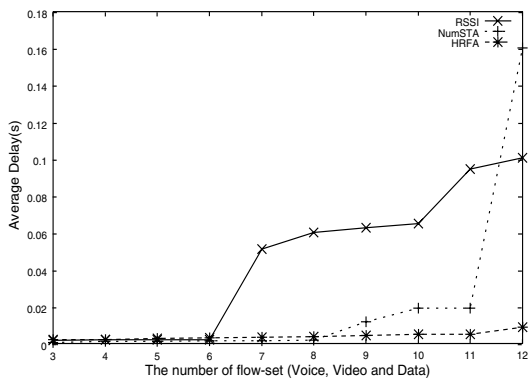

Fig. 11. Average delay of voice traffic in Case2

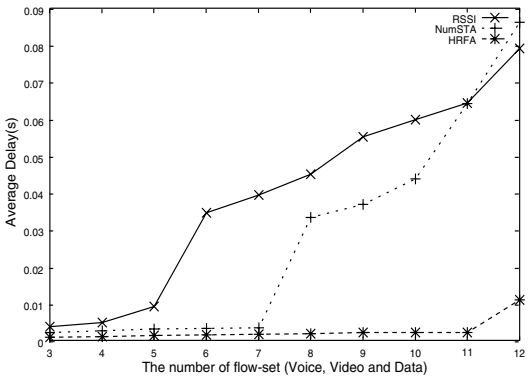

Fig. 12. Average delay of video traffic in Case2

rate which a STA uses to communicate with an AP and traffic load in the AP. Thus it achieved both load balancing and the efficient use of radio resource, and it also obtained higher througput compared to other schemes. Since HRFA is evaluated in a simple WLAN network in this paper, we will further evaluate the performance of HRFA when it is used in a WLAN network which is dynamically changing and when some $\mathrm{HO}$ algorithm is applied to HRFA.

\section{REFERENCES}

[1] "Wireless LAN Medium Access Control (MAC) and Physical Layer (PHY) Specifications", IEEE Std 802.11 -1999 (Reaff 2003).

[2] "Wireless LAN Medium Access Control (MAC) and Physical Layer (PHY) Specifications: Medium Access Control (MAC) Quality of Service (QoS) Enhancements", IEEE Std. 802.11e/D8.0, Feb. 2004.

[3] "Wireless LAN Medium Access Control (MAC) and Physical Layer (PHY) Specifications: Higher-Speed Physical Layer Extension in the 2.4 GHz Band", IEEE Std. 802.11b-1999, 1999.

[4] M. Kappes, A.S. Krishnakumar and P. Krishnan, "Estimating Signal Strength Coverage for a Wireless Access Point", in Proc. of IEEE Globecom 2004, Nov. 2004.

[5] A. Balachandran, P. Bahl and G.M. Voelker, "Hop-Spot Congestion Relief in Public-area Wireless Networks," in Proc. of WMCSA, June 2002.

[6] Y. Xiao, H. Li, and S. Choi, "Protection and Guarantee for Voice and Video Traffic in IEEE 802.11e Wireless LANs", in Proc. of IEEE INFOCOM 2004, March 2004.

[7] I. Ramani and S. Savage, "SyncScan: Practical Fast Handoff for 802.11 Infrastructure Networks", in Proc. of IEEE INFOCOM 2005, March 2005

[8] I. Papanikos and M. Logothetis, "A study on dynamic load balance for IEEE 802.11b wireless LAN," in Proc. of COMCON 2001, 2001.

[9] Y. Fukuda and Y. Oie, "Decentralized Access Point Selection Architecture for Wireless LANs -Deployability and Robustness", in Proc. of IEEE VTC2004-fall, Sep. 2004.

[10] K. Saitoh, Y. Inoue, M. Iizuka and M. Morikura, "An Effective Data Transfer Method By Integrating Priority Control into Multirate Mechanisms for IEEE802.11 Wireless LANs", in Proc. of IEEE VTC2002spring, March 2002.

[11] J.D.P. Pavon and S. Choi, "Link Adaptation Strategy for IEEE802.11 WLAN via Received Signal Strength Measurement", in Proc. of IEEE ICC2003, June 2003.

[12] "http://www.isi.edu/nsnam/ns/index.html" 